\title{
Metrology of Non-Rigid Objects
}

Kenneth L. Blaedel, LLNL

David W. Smith, LLNL

Andre A. Claudet, LLNL

Eric P. Kasper, Cal-Poly State University

Steven R. Patterson, UNC-C

\section{January 2002}

U.S. Department of Energy

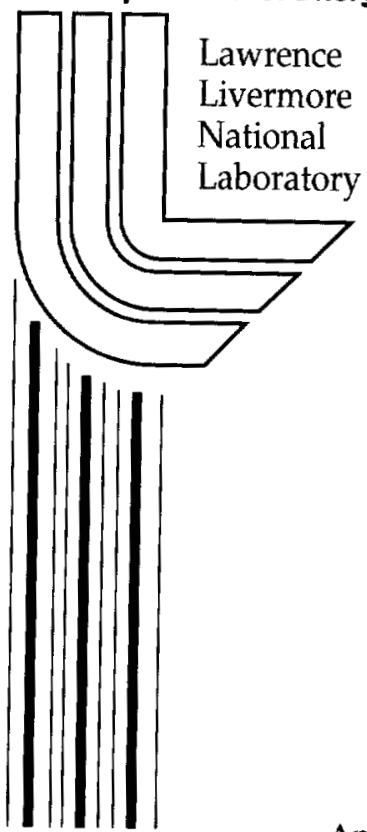


This document was prepared as an account of work sponsored by an agency of the United States Government. Neither the United States Government nor the University of California nor any of their employees, makes any warranty, express or implied, or assumes any legal liability or responsibility for the accuracy, completeness, or usefulness of any information, apparatus, product, or process disclosed, or represents that its use would not infringe privately owned rights. Reference herein to any specific commercial product, process, or service by trade name, trademark, manufacturer, or otherwise, does not necessarily constitute or imply its endorsement, recommendation, or favoring by the United States Government or the University of California. The views and opinions of authors expressed herein do not necessarily state or reflect those of the United States Government or the University of California, and shall not be used for advertising of product endorsement purposes.

This work was performed under the auspices of the U.S. Department of Energy by the University of California, Lawrence Livermore National Laboratory under Contract No. W-7405-Eng-48.

This report has been reproduced directly from the best available copy.

Available to DOE and DOE contractors from the Office of Scientific and Technical Information P.O. Box 62, Oak Ridge, TN 37831

Prices available from (423) 576-8401 http://apollo.osti.gov/bridge/

Available to the public from the National Technical Information Service

U.S. Department of Commerce 5285 Port Royal Rd., Springfield, VA 22161 http://www.ntis.gov/

OR

Lawrence Livermore National Laboratory Technical Information Department's Digital Library http://www.llnl.gov/tid/Library.html 


\section{Problem Definition}

Dimensional characterization of non-rigid parts presents many challenges. For example, when a non-rigid part is mounted in an inspection apparatus the effects of fixturing constraints cause significant deformation of the part. If the part is not used in normal service with the same load conditions as during inspection, the dimensional characteristics in service will deviate from the reported values during inspection. Further, the solution of designing specialized fixturing to duplicate "as-installed" conditions does not fully resolve the problem because each inspection requires its own methodology. The goal of this project is to formulate the research problem and propose a method of assessing the dimensional characteristics of non-rigid parts.

The measured dimension of a rigid component is traceable at some level of confidence to a single source (NIST in the USA). Hence the measurement of one component of an assembly can be related to the measurement of another component of that assembly. There is no generalized analog to this pedigreed process for dimensionally characterizing non-rigid bodies. For example, a measurement made on a sheet-metal automobile fender is heavily influenced by how it is held during the measurement making it difficult to determine how well that fender will assemble to the rest of the (non-rigid) car body. This problem is often overcome for specific manufacturing problems by constructing rigid fixtures that over-constrain the non-rigid parts to be assembled and then performing the dimensional measurement of the contour of each component to check whether each meets specification. Note that such inspection measurements will yield only an approximation to the assembled shape, which is a function of both the geometry and the compliance of the component parts of the assembly. As a result, non-rigid components are more difficult to specify and inspect and therefore are more difficult to purchase from outside vendors compared to rigid components. The problems are compounded as the requirements come to include higher and higher precision.

The central idea for this project is the concept of a "free shape." The free shape is the geometry of the part when no loads are present. That is, when those loads produced by fixturing, gravity and others are not present. Since it is impossible to directly measure the free shape, some method for inferring it must be developed. Once the free shape is known some metric must be developed for acceptance or rejection of the part.

\section{Applications}

The metrology of non-rigid parts is of direct interest to the laboratory in at least three ways. First, it has implications concerning the characterization of thin, hemispherical shells ("hemi-shells"). Second, it has application to the metrology of thin photomasks for extreme-ultraviolet lithography (EUVL). Third, it can help in the inspection of potassium dihydrogen phosphate (KDP) crystals in advanced laser systems. It is important to industry because it has applications to metrology of sheet-metal parts.

\section{Inspection of Hemi-Shells}

The Stockpile Stewardship Program demands a better understanding of the deformation that occurs in hemi-shells resulting from their manufacture and assembly. Hemi-shells are non-rigid bodies, which for example, deform under their own weight and are necessarily deformed by fixtures that hold them during the manufacturing process. In 
order to inspect a hemi-shell, it is constrained in a fixture that "rounds up" the equator of the shell and thus purposely removes some of the deformation of the shell that results, for example, from non-uniform residual stress. The reason for using the rounding-ring fixture is that this places the hemi-shell under a condition like it would take upon assembly to its mating hemi-shell. Under this condition, measuring the outer contour is a closer estimate to the contour taken upon assembly than measuring the outer contour with the part supported in a fixture that constrains only the six rigid-body motions.

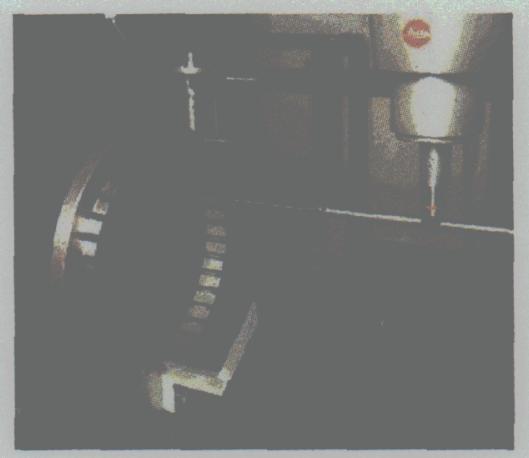

Figure 1: Over-constraining Fixture

Figure 1 illustrates a rounding-ring fixture. The inspector can then compare the measurement of the constrained shell and the drawing of a perfect shell and determine, within some uncertainty, whether it is acceptable or not. However, a number of problems arise from using such fixtures. One is that the fixture, which over-constrains the shell, imposes ill-defined constraints on the shell. Hence, slight variation in the procedure or conditions of the measurement introduces uncertainty into the dimensional measurement. In addition, the fixturing and measurement processes that have arisen at the different agencies within the DOE complex are unique to each agency and the measurement data are not traceable from one institution to another.

\section{Photomasks for Extreme Ultraviolet Lithography (EUVL)}

Photomasks for EUVL are thin reflective plates with patterns of integrated circuits that are to be imaged onto silicon wafers. The budget for image placement error for EUV Lithography dictates that the patterned surface of the photomask depart less than $100 \mathrm{~nm}$ from a flat plane as it is mounted horizontally in the lithographic exposure tool. 


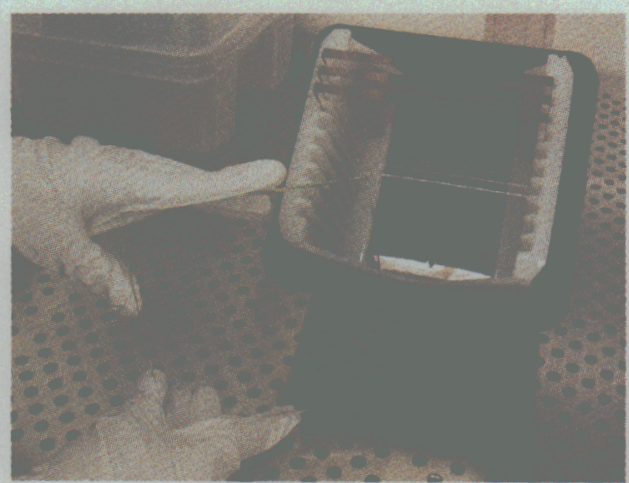

Figure 2: Photomask Substrate

Unfortunately, the photomasks have an aspect ratio of edge-length to thickness of about 25 and are subject to deformation by very small external forces (Figure 2). For example, the gravity-induced sag of a photomask on three-point support is approximately ten times the allowable departure from a flat plane. Thus the mask must be deformed into being flat as it is mounted in the exposure tool. This therefore gives rise to the problem of how to specify and inspect photomasks so as to ensure that it can be brought into a flat state as it is mounted in the exposure tool. The current standard (SEMI P37) allows only a 50-nm P-V departure from flatness for the freestanding photomask. Thus, the current standard takes a very conservative approach by treating the photomask like a rigid body, that is, that the chuck has no ability to flatten the mask and any existing error in the freestanding photomask appears directly in the as-mounted condition. While the current specification does guarantee that the photomask will perform, producing photomasks to specification is difficult and expensive. A relaxed specification of the free-shape that still guarantees that a photomask can be brought into specified is desirable.

\section{KDP Crystals}

Thin, high precision KDP crystals are used in NIF. Crystals are manufactured in the constrained condition. The final thickness of the finished crystal is approximately $1.0 \mathrm{~cm}$. With a width and height of $42 \mathrm{~cm}, \mathrm{KDP}$ crystals have an aspect ratio that makes them very non-rigid (Figure 3 ).

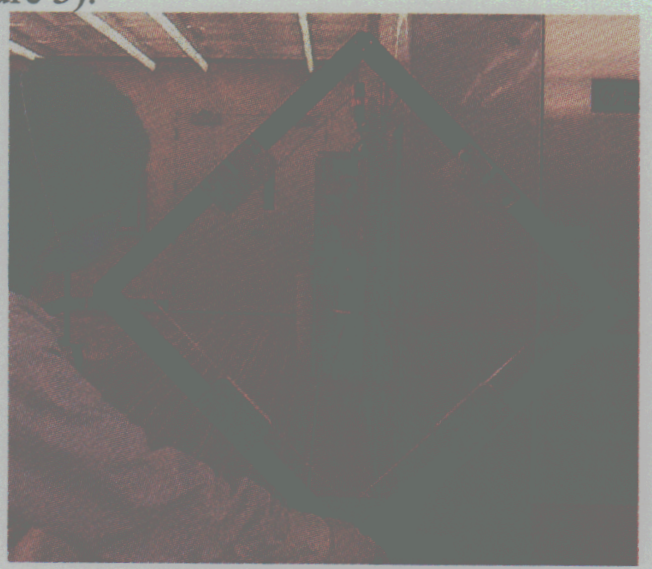

Figure 3: KDP Crystal in Holder 
The finished crystals are inspected in a vertical, freestanding condition using optical techniques. The crystals are then edge constrained for use at a variety of angles relative to gravity. The combined effects of gravity loading and constraint induced errors turn out to be one of the dominant sources of error affecting the performance of the crystal. Extensive modeling and analysis has been performed to better understand these effects while attempting to improve on them in the mechanical design.

A method for assessing the constraint system and the influence of gravity and other boundary conditions would greatly improve the ability to predict and/or improve performance of NIF KDP crystals.

\title{
Other Applications
}

Sheet metal parts are a common example of non-rigid parts. For example, automotive body panels must have precise geometry for aesthetic reasons. Elaborate, specialized fixturing devices and metrics are used for characterization. A generalized method for assessing the dimensional characteristics of the parts would provide for a more flexible metrology process.

One design goal for space-based optics is to minimize mass. The optics must be lightweight to facilitate transportation into orbit. Thus, the option of adding material to increase rigidity is a very expensive solution. The design exercise is to manufacture and measure an optic under gravity and accurately predict the shape it will take in space. If there is large uncertainty associated with the predicted shape, then additional stiffness, and its concomitant increase in mass is incorporated into the design to reduce the uncertainty of predicting the shape in space.

\section{Technical Approach}

The examples of the hemishells and the photomasks above illustrate two current approaches to measuring and specifying non-rigid parts. In the case of the hemishells, which is illustrated in Figure 4, they are measured in a rigid fixture that approximates the functional environment of the part and the measurement is directly compared to a drawing of the ideal part to decide on the acceptability of the part.

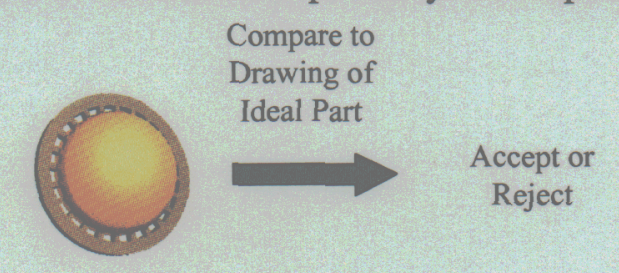

\begin{abstract}
Inspection data taken under constraint set approximating functional shape.
\end{abstract}

Figure 4: Hemishell Inspection Process

With this approach it may be very difficult to know and control the constraints that the fixture imposes on the part. Hence, the fixtured state becomes a poor approximation to the state that the part will see in service. For example, if the part were to be assembled to another non-rigid part, then a stiff fixture would poorly approximate the assemble condition. In addition, the shape of a non-rigid part can change depending 
on the order in which it is placed into the constraints of the fixture, which introduces uncertainty in the inspection process.

In the case of the photomasks, which is illustrated in Figure 5, they are measured in a state that approximates the free-state and the free-state measurement is directly compared to a drawing of the ideal part.

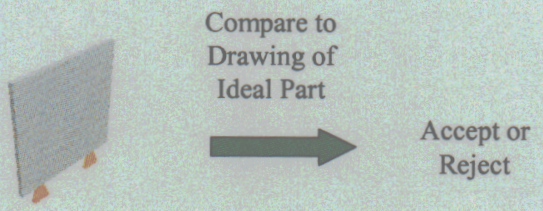

$$
\begin{aligned}
& \text { Inspection data taken under } \\
& \text { constraint set approximating } \\
& \text { free-shape. }
\end{aligned}
$$

Figure 5: Photomask Inspection Process

Here, the free state is a very poor approximation to the state that the part will see in service. In essence, the inspection process of photomasks is much the same as that for a rigid body. While these two approaches to inspecting hemishells and photomasks appear quite similar, they lead to very different inspection fixtures.

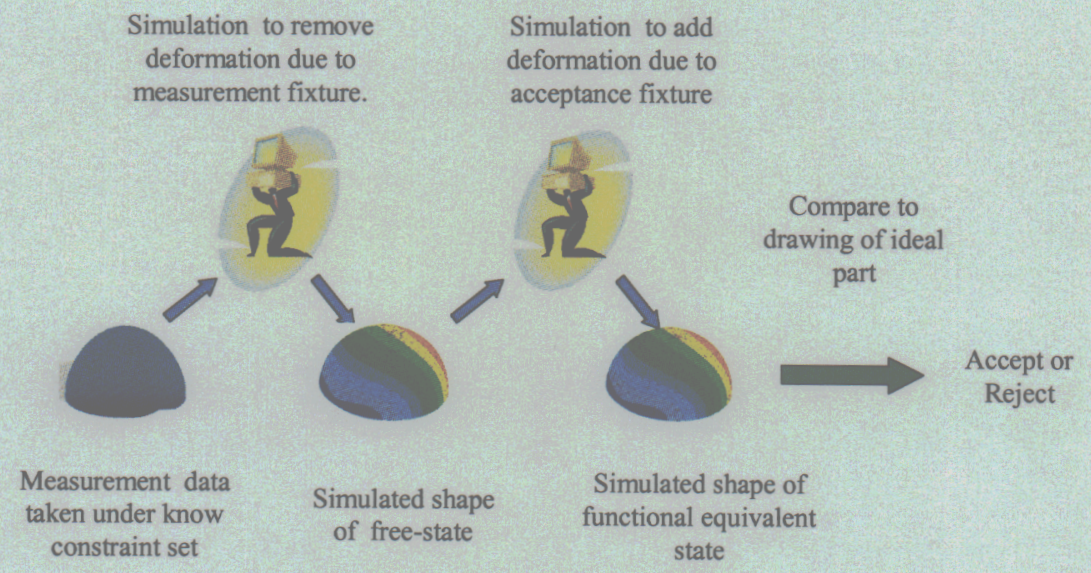

Figure 6: Technical Approach

A new method of inspecting non-rigid bodies is proposed and developed here. As shown in Figure 6, it begins with a measurement of the non-rigid part as it is constrained under well-defined conditions, which may be quite different from the fixtures used in the two examples above. The deformation imposed by the well-defined constraints can then be removed by simulation to predict the shape of the part as though it were free from external forces. This "free-state" condition, which is not physically realizable, is then input into a second, acceptance, fixture that simulates the functional state, which is then compared to a drawing of the ideal part to decide on the acceptability of the component part. The first advantage of this method is that separates the two functions that the single fixture must perform in the previous cases. The single important criterion for the measurement fixture is that it allows the external constraints to be exactly known. The 
single important criterion for the acceptance fixture is that it simulates the functional environment. But, because the acceptance fixture is a transformation of the measured data, it can be embodied as a finite element simulation that represents the part's response to an ideal fixture. The simulation is free from problems like friction between the part and a hardware fixture that over-constrains the part.

Consider first the hemispherical shell ("hemishell") described above, whose outer contour is required to be spherical to some tolerance when it is assembled to a hemispherical mate. In current practice, a hemishell is inspected by forcing the equator to be round in an inspection fixture ("rounding ring"), then the diameter is measured and the spherical contour is measured in this constrained state. In essence, the rounding ring is used to bring the part into its approximate functional state. As described previously, the problem with using a rounding ring is that it introduces unknown constraint conditions on the part that are quite different from what it may see in assembly. For example, the process of forcing the rounding ring over the equator tends to trap friction between rounding ring and hemishell, which results in significant deformation of the spherical contour. Note that the deformation occurs not just at the equator where the rounding ring contacts the hemishell, but propagates quite far from the equator. Thus a perfect part may be rejected because of the friction associated with using the rounding ring. Also, any mismatch in the circumference of the rounding ring and the length of the equator of the part causes significant radial forces and moments at the equator and concomitant deformation of the spherical contour.

The new approach is to use a measurement fixture that allows the free-state shape to be calculated and then to use an "acceptance" fixture that simulates its functional state. The acceptance fixture, which is a structural finite element analysis, does not suffer a number of the shortcomings of the hard fixture. The acceptance fixture simply represents a hard fixture that is free of friction between the fixture and part free of any mismatch between the length of the equator of the part and the circumference of the fixture.

Consider a second case, the photomasks described above, which are required to be flat to some tolerance as they are sequentially mounted in an exposure tool. In this case, requiring the free-state shape of the photomask to be flat is overly restrictive because the mounting process has some capability to flatten the mask. One such mounting method is to electrostatically clamp the photomask to a planar surface. Because a photomask is almost planar, it can be measured with its patterned surface in a vertical plane and thus the deformation due to gravity becomes extremely small. Hence, the measured state is very close to the free-state shape. (This is unlike the hemishells, which sag under gravity regardless of how they are oriented or fixtured.)

One method of inspecting photomasks is to mount them to an inspection fixture, one that replicates the actual chuck in the exposure tool, and then measure the resultant flatness. As with the hemishell inspection, the inspection fixture suffers from practical shortcomings. An example is that if a particle becomes trapped between the chuck and the photomask, then the deformation that is caused by the particle would cause the rejection of a good photomask.

The new approach is again to use an acceptance fixture, which operates on the free state measurement, and which is a model of the flattening ability of the chuck. In this case, a structural analysis is performed of constant pressure acting to deform the photomask against a rigid, flat plane. Applying this model to a the measurement of the 
freestanding photomask allows the inspector to determine whether the mask will be flattened within specification, or whether its freestanding state is so badly deformed that it cannot be flattened.

As proposed, the new method requires that an analysis be performed for each part that is to be inspected. However, the number of simulations can be radically reduced if desirable and ease the job of deciding whether to accept or reject a particular part. For example, a more practical approach is to decompose the errors in the freestanding part, that is, the departure of the freestanding part from the ideal part, and then perform the analysis on the decomposition of the error. In this way, the analysis need only be applied to each mode of the decomposition to understand how that mode is contributes to the predicted functional shape. The analysis is therefore performed only once for each mode, in advance of all inspection measurements.

A further simplification brought about by decomposing the error of the freestanding part into modes is that the specification process to describe an acceptable part can be performed in terms of these modes. In the case of the photomasks for example, the departure of the freestanding shape from a flat plane is decomposed into orthogonal Legendre modes. The electrostatic chuck can easily flatten the low-order modes, but does little to attenuate higher-order modes. Therefore, a part can contain higher amplitudes of low-order modes and still be quite acceptable. The specification states exactly what amplitude of each mode is acceptable. This changes the acceptance decision from one of directly comparing the difference between the part measurements to a drawing to one of assessing the magnitudes of modes in the decomposition of the error. This is analogous to limiting the power spectrum of the error of an optical surface as a way to specify its acceptability.

In special cases, additional simplification may arise in determining the acceptance of a part. Other criteria can be used to determine the acceptability of a part such as the energy to deform the freestanding part into the ideal size and shape. Another criterion is the maximum stress that occurs in deforming the freestanding part into the ideal part.

\section{Method of Modeling the Deformation Due to Fixturing}

\section{Finite Element Analysis}

Finite element method is the simplest way to model and simulate the deformations of thin shells subject to various boundary conditions. The models can be built using various elements described in the previous sections. Commercial and homemade finite element codes are used to simulate the results for thin shell problems in this project. The commercial codes considered for use on this project are Pro Mechanica, ANSYS, ABAQUS, and COSMOS. The homemade finite element codes considered for this project are Nike, and FEAP (a simple Fortran FEA code). Nike is a finite element code that has been developed for internal use at Lawrence Livermore National Laboratory. These codes are used to check and verify the consistency of the results of each code relative to another. Pro Mechanica, ABAQUS, COSMOS, and FEAP were selected for use on this project. 


\section{Close Form Solution}

Closed form solutions were also evaluated for this project. The selection of load cases for the actual experiment does not have closed form solutions but simpler load cases were evaluated and compared to the results of the FEA. An example of a simple load case that has a closed form solution is a cylinder with a uniform pressure applied to the inner or outer surface. Comparison of results between this simple load case and the FEA results allowed the experimenters to eliminate Pro Mechanica as a viable option for this project. Pro Mechanica produced results that were inconsistent with closed form results and the other FEA codes.

\section{Uncertainty in the Process}

In order to increase the usefulness of reported measurement values a statement of uncertainty must be included. Thus, the many contributors to the overall uncertainty must be assessed. In addition to the typical uncertainties associated with data acquisition (hardware), there is additional uncertainty associated with the FEA. A "home grown" FEA code was used for this work. Thus, more complete knowledge of the underlying operations and the associated uncertainty was realized. Significant contributors to the overall uncertainty are given below.

\section{Material Properties}

Assigning material properties to an element in an FEA study makes the significant assumption that the part model being analyzed has the same material property as the actual part. It is typically assumed that the parts will be homogeneous and isotropic. In most instances these assumptions are not completely accurate. These assumptions result in uncertainty in the FEA results. The effect of variation in the material properties on the FEA results can be determined. For example, the Young's modulus is directly proportional to deflection. Thus, if the Young's Modulus varies by $20 \%$, then deflections can also vary by $20 \%$.

Standard tensile tests can accurately measure the Young's modulus of a material to about $1 \%$. The accuracy is worse for determining Poisson's ratio as a result of the uncertainties in measuring lateral displacement. Ultrasonic methods for measuring mechanical properties have been explored and results show improved accuracy in the determination of mechanical properties. The fundamental principle is measurement of ultrasonic velocities generated by a dynamic pressure wave into a material of known thickness and measuring the transit time of the emerging acoustic pressure wave. The uncertainty in Young's modulus calculation based on this based is dependent on the density of the material and the thickness of the medium. The Young's modulus' calculated uncertainty based on uncertainties in thickness and density is determined to be around $0.4 \%$. This uncertainty is improved to $0.2 \%$ with improved density measurement. Detailed results for this calculation are given in Appendix A.

\section{Mesh}

The accuracy of the solution is dependent on the quality of the mesh. The FEA convergence is also greatly affected by the mesh quality. The global displacement should converge to a stable value and any results of interest should converge locally. A more 
subjective measure of the quality of the mesh is its appearance and ability to visually convey the geometry it represents. Typically, the better-looking mesh is better and a badlooking mesh almost always indicates a problem. Equilateral triangles and squares are the ideal elements to use with smooth and gradual transitions without skinny, distorted elements. The type of elements used (i.e. p-elements or h-elements) is also a factor in mesh density. The use of higher order p-elements does not require as dense a mesh as same h-elements for the same model. P-elements allow for higher edge polynomial orders which can improves the representation of the load curvature.

\section{Boundary Conditions}

Boundary conditions are difficult to model since physical interactions of constraints and modeled geometry are complex. For the purposes of experimental validation it is possible to design geometry and fixtures to create boundary conditions that are more accurately modeled. This has been achieved by producing boundary conditions that have very little friction. An exact constraint fixture also greatly reduces the unknown forces acting on the model.

\section{Linear Static Assumption}

Linear static solutions are most common solutions available when using finite element analysis. The popularity of this solution often obscures the fact that it represents a significant assumption of linear events. Linear event are typical idealized in most problems and do not typically exist. However, linearity in thin shell analysis is a good assumption because of low-stress condition that is created during bending of the shell. Thin shells are used to experimentally validate the process for this work.

\section{Element Type}

When using finite element analysis different element types can be used to model thin shells. Two candidate elements for this work are shell elements and hexahedral solid elements. Shell elements typically represent thin-walled structures. They can be quadrilateral or trilateral. A quadrilateral mesh is usually more accurate mesh of similar density based on trilateral elements. Most preprocessors can mesh a surface with quads only or apply a quad dominant mesh where triangles are used only when the mesher cannot resolve an area within specified element tolerances. Triangles are acceptable in regions of gradual transitions. Linear or first order shell elements are normally planar and degrade in accuracy as their initial definition deviates from planar. This is an issue only for quad elements because a three-noded triangle must be planar. Higher order shell elements can provide accurate results with curved initial geometries. A benefit for using higher order elements is that positioning the mid-side nodes on the actual curved geometry increases the model's accuracy. P-elements are ideal to represent bi-directional curvature and can smoothly represent initial geometry.

Most first order triangle element are only capable of calculating a single strain value across the entire element. Consequently, they are known as constant strain elements. This limitation can lead to overly stiff results under a given load as localized strain gradient will be difficult, if not impossible, to capture. They do provide adequate results when used on flat or gently curving surfaces with minimal strain variance across the span. Linear quad elements have a linear strain distribution from one node to the next 
so they are better at capturing localized stresses. Adding mid-side nodes to both these elements improves their strain distribution by a polynomial order.

\section{III-Condition Stiffness Matrix}

Ill-condition stiffness matrix results in solutions that could be an order of magnitude different in value from a few percent change in the stiffness coefficient matrix. In matrix terminology, the rows of an ill-conditioned matrix are almost linearly dependent. For a 2 by 2 systems, this means that the second row of the coefficient matrix is almost a scalar multiple of the first row. In structural terminology, a major cause of illconditioning in practical finite element models is a large different in stiffness with the stiffer region being supported by the more flexible region. This circumstance shifts essential numerical information to the latter digits of stiffness coefficient Kij. These latter digits may be so few in number that the solution in worthless. Physically, the stiffer region has one or more displacement states that are almost rigid-body motions within a more flexible supporting structure. The limiting case in a structure without any supports: it has only rigid-body motion in static analysis, and its stiffness matrix is singular.

In this particular study this problem can be encountered when a flexible shell element is modeled and it is connected to a stiff boundary condition. This can result in high unrealistic deflections at the node. This problem can be mitigated by distributing the loading at number of nodes around the boundary condition as oppose to a single node connection.

A numerical measure of ill-conditioning in a coefficient matrix is the condition number. A large condition number denotes an unstable solution and warns that a finite element solution may contain appreciable error. The condition number of matrix $\mathrm{K}$ (stiffness matrix) is simply defined as (1),

$$
C(\mathrm{~K})=\frac{\lambda_{\max }}{\lambda_{\min }}
$$

where $\lambda_{\max }$ and $\lambda_{\min }$ are the largest and smallest eigenvalues of matrix $K$. It can be shown that for each power of ten in the ratio $\lambda_{\max } / \lambda_{\min }$, the operations of equation solving lose about one digit of accuracy in the displacement mode associated with $\lambda_{\min }$ (Cook, 1989). The estimated accuracy loss is (2).

$$
\text { accurate digit loss } \approx \log _{10} \frac{\lambda_{\max }}{\lambda_{\min }}=\log _{10} C .
$$

For example, for $\mathrm{C}(\mathrm{K})=105$, if computer has seven-digit capacity, only two digits are reliable in the computed displacements. With sixteen-digit capacity, eleven accurate digits remain.

\section{Uncertainty Management}

An effective measurement requires management of uncertainty. Once the uncertainty contributors are identified steps can be taken to minimize their magnitude. For this work action is required on both the data acquisition (hardware) and analysis 
(software) sides. On the hardware side, multiple measurements are taken and the fixturing forces and displacements are recorded. To facilitate the analysis, the cylinder is measured close to its free shape the material properties are measured.

Table 1: Uncertainty Contributors

\begin{tabular}{|l|c|l|}
\hline \multicolumn{1}{|c|}{ Source of Uncertainty } & Magnitude of Uncertainty & \multicolumn{1}{c|}{ Remedy } \\
\hline Cylinder wall de-center & $2 \mu \mathrm{m}$ & Measure surfaces independently \\
\hline Axial coordinate measurement & $0.5 \mathrm{~mm}$ & Use different measuring machine \\
\hline Idealized vs. Exact Geometry & $8 \mathrm{parts}$ in 100 & Use "Exact" geometry \\
\hline Element Domain - $\mathrm{n}$ Flat Facets & $1-\cos (\mathrm{pi} / 4 \mathrm{n})=1 \mathrm{e}-7$ & Use Curved Elements for $\mathrm{n}>36$ \\
\hline Mesh Properties & $\mathrm{Log}(\mathrm{C} /[\mathrm{K}])$ & Avoid Ill-Conditioned K-Matrix \\
\hline Localized Contact at Boundary & 14 parts in 100 & Exclude Local Contact Area \\
\hline Isotropic Material Properties & 4 parts in 1000 & Improve Material Testing \\
\hline Material Orthotropy & Moderate & Improve Material Testing \\
\hline Element Type Selected & Moderate & Select Problem Specific Elements \\
\hline Numerical Integration & Zero & Linear Map Elements to Model Space \\
\hline
\end{tabular}

Table 1 illustrates some uncertainty contributors and suggestions for remedying them.

\section{Experimental Demonstration}

A copper thin walled cylinder was manufactured under carefully controlled conditions. A cylinder was chosen because of its manufacturability and because it can be oriented so that gravity has little effect. The goal of the design was to reduce the uncertainty in the measurement to small values compared to those for FEA.

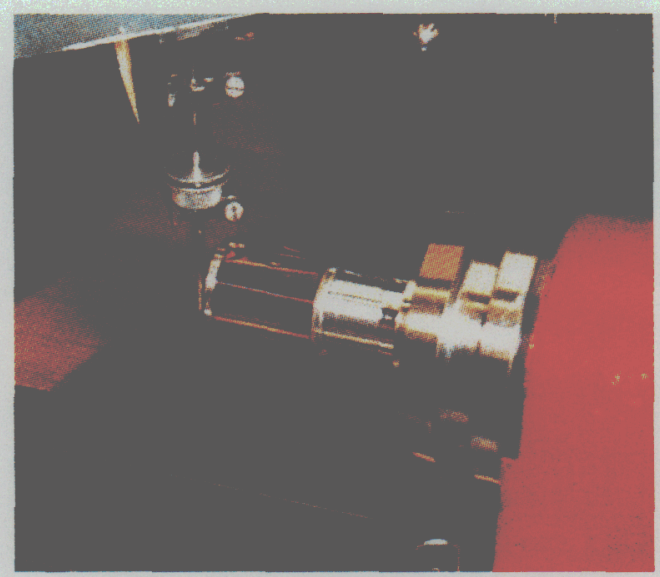

Figure 7: Test Cylinder Manufacturing

Figure 7 shows the cylinder at an intermediate step in the manufacturing process. A fixturing device was built to exactly constrain the cylinder. The design facilitated readings of both displacements of the cylinder and reaction forces at the constraints. 


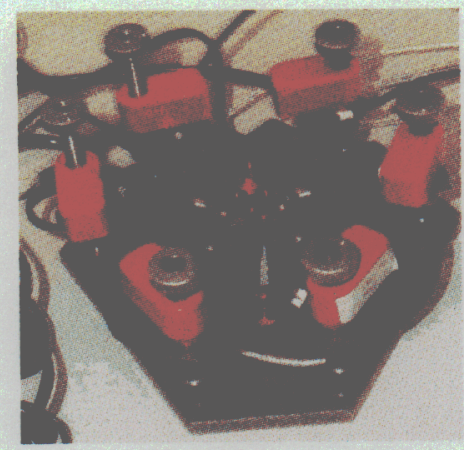

Figure 8: Test Artifact Fixture

The actuators on the fixturing device deformed the cylinder approximately $2 \%$ of its wall thickness over 15 steps. Measurements of the displacement were obtained by placing the entire fixturing device on a Tropel CylinderMaster 25 cylinder inspection device.

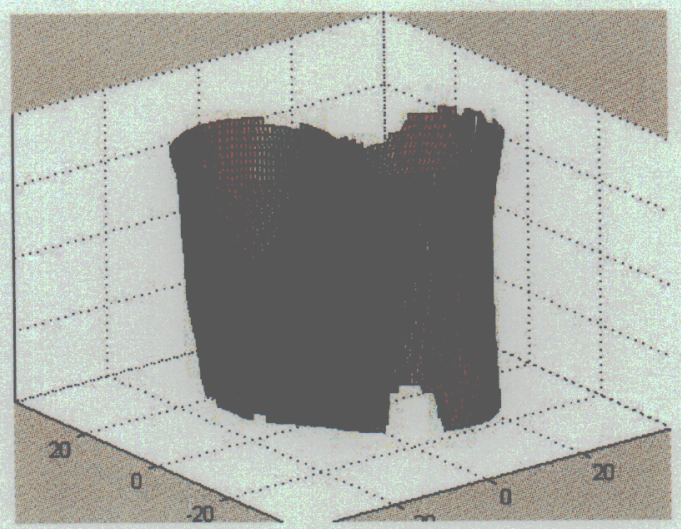

Figure 9: Exaggerated Deformations

Figure 9 illustrates the deformations (exaggerated) induced by the fixture. The fixture contacted the cylinder at 3 points and produces a tri-lobe deformation.

\section{Measurement Data Analysis}

The goal of the numerical simulation section is to determine the stress-free configuration for the test cylinder from a given set of experimental data. The experimental data was determined based on a set of sequential optical measurements. The experimental data was determined follows: first, the test cylinder was set on a loading fixture and an initial radial deviation map is obtained, denoted as $\Delta \mathrm{X}_{0}$. The test cylinder was then incrementally displaced at three points on the outer surface of the cylinder and the corresponding radial deviations are recorded. A maximum radial displacement of approximately $2 \%$ of the wall thickness is imposed over 15 increments. 


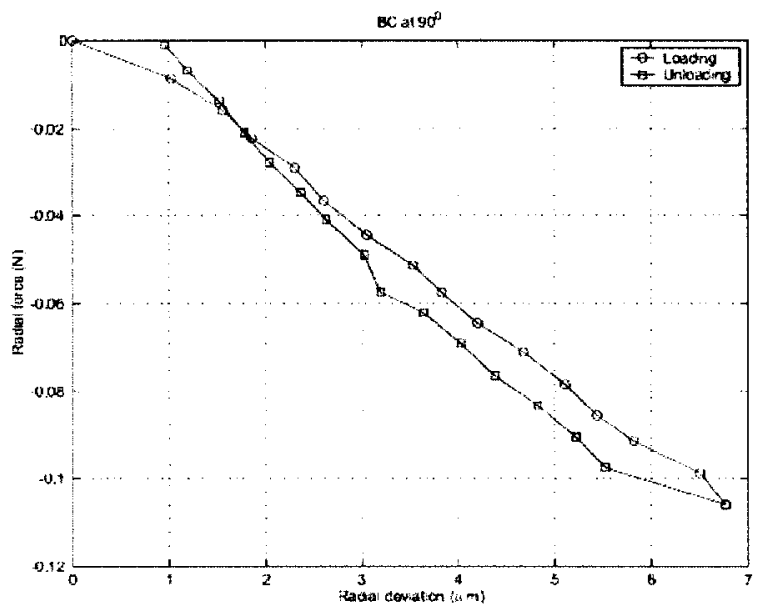

Figure 10: Load-Displacement Curve at $90^{\circ}$ Displacement Boundary Condition

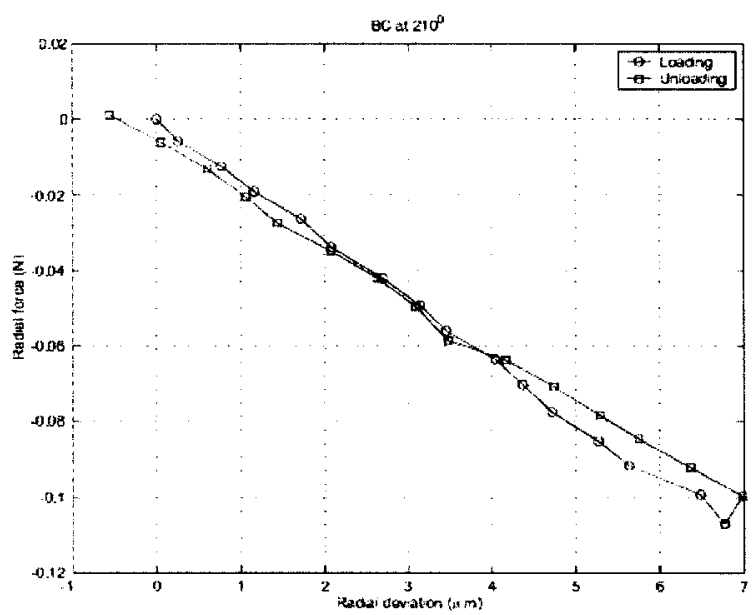

Figure 11: Load-Displacement Curve at $210^{\circ}$ Displacement Boundary Condition

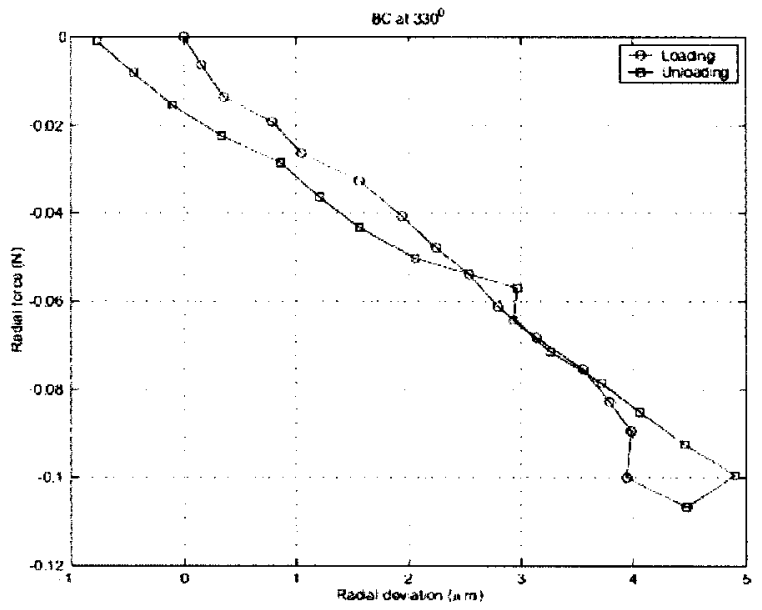

Figure 12 Load-Displacement Curve at $330^{\circ}$ Displacement Boundary Condition 
A plot of the radial force versus radial displacement for each of the three boundary conditions is shown in Figure 10 through Figure 12. As shown from Figure 10 through Figure 12 the response is linear and returns to its initial state to within the uncertainty of the optical measurements. Given the linearity and the inherent elasticity of the load-deflection curves a linear-elastic shell formulation was used for the numerical simulations. In addition, it is also assumed that the elastic material properties and wall thickness are known and constant throughout the deformation.

An overview of the procedure used to determine the stress-free state is given below.

- The initial finite element discretization was determined by superimposing the experimental radial deviation map in the unloaded configuration, $\Delta \mathrm{X}_{0}$, to the geometry of a "pure" right circular cylinder, $\mathrm{X}_{0}$. The resulting initial finite element discretization is denoted as $\mathrm{X}=\mathrm{X}_{0}+\Delta \mathrm{X}_{0}$.

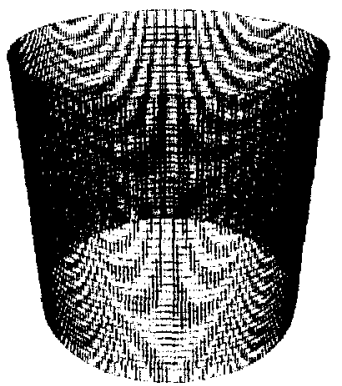

Figure 13 Finite Element Mesh Discretization, $256 \times 35$

Note the grid resolution of the experimental data and the finite element discretization coincide, specifically the grid consists of 256 data points around the circumference and 35 data points along the longitudinal axis of the cylinder, see Figure 13.

- Since the discretization of the experimental data and the finite element mesh coincide the prescribed normal displacement boundary conditions correlate directly to the experimental data.

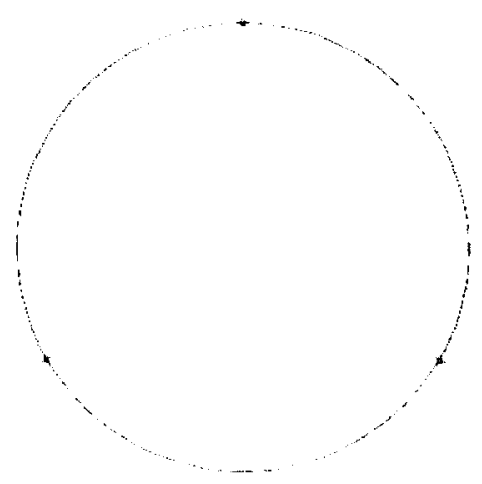

Figure 14: Locations of the Radial Boundary Conditions 
The loading fixture imposes a prescribed normal displacement (over 15 increments), of approximately $2 \%$ of the wall thickness, to the outer surface of the cylinder at three locations equally spaced. Figure 14 illustrates the radial displacement boundary conditions.

- Given the initial finite element discretization, $\mathrm{X}$, and the three displacement boundary conditions (based on the experimental data) contour maps of the radial displacement for the experimental and a preliminary finite element simulation can be plotted.
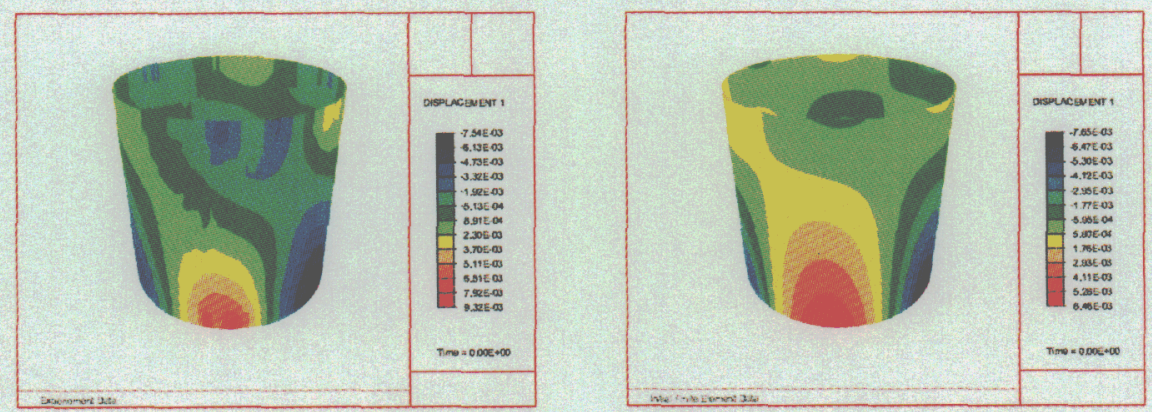

Figure 15: Contour Plot of Radial Displacementsfor the Experimental Data and Initial Finite Element Discretization. Units are $\mathrm{mm}$.

Figure 15 shows the contour maps. Note that both the experimental and numerical results are based on the same initial configuration.

- Recall the goal is to minimize the error between the deformed coordinates of the finite element discretization to that of the experimental data. To obtain this minimization a simple coordinate update scheme shown below is utilized.

1. Initialize iteration counter $\mathrm{k}=0$

2. Set current coordinates $\mathrm{x}^{(\mathrm{k}+1)}=\mathrm{X}$

3. Solve for the nodal displacements $\mathrm{u}^{(\mathrm{k}+1)}$,

$$
u^{(k+1)}=K^{-1}\left(x^{(k+1)}\right) R,
$$

where $\mathrm{K}$ is the tangent matrix and $\mathrm{R}$ is the residual vector.

4. Update iteration counter $\mathrm{k}=\mathrm{k}+1$

5. Update the coordinates by setting $x^{(k+1)}=x^{(k)}+\left(\Delta X-u^{(k)}\right)$ where $\Delta X$ is the difference in the initial and final configurations (loading portion of the curve) of the experimental data.

6. Solve for the displacements, $u^{(k+1)}$, as 


$$
u^{(k+1)}=K^{-1}\left(x^{(k+1)}\right) R
$$

7. Check for convergence

$$
r=\left\|u^{(k+1)}-u^{(k)}\right\|
$$

8. If ( $r>T O L)$ repeat from (4) or else finish and report $x=u^{(k+1)}$

- Once convergence is achieved then the updated coordinates, $x$, are stored. Note using an initial finite element discretization with coordinates, $\mathrm{x}$, and imposing the experimental displacement boundary conditions the final deformed configuration of the numerical model will approximate the deformed configuration of the experimental data to within the uncertainty of the data. The stress-free configuration is then obtained by unloading the radial displacement boundary conditions or simply $\mathrm{x}$.
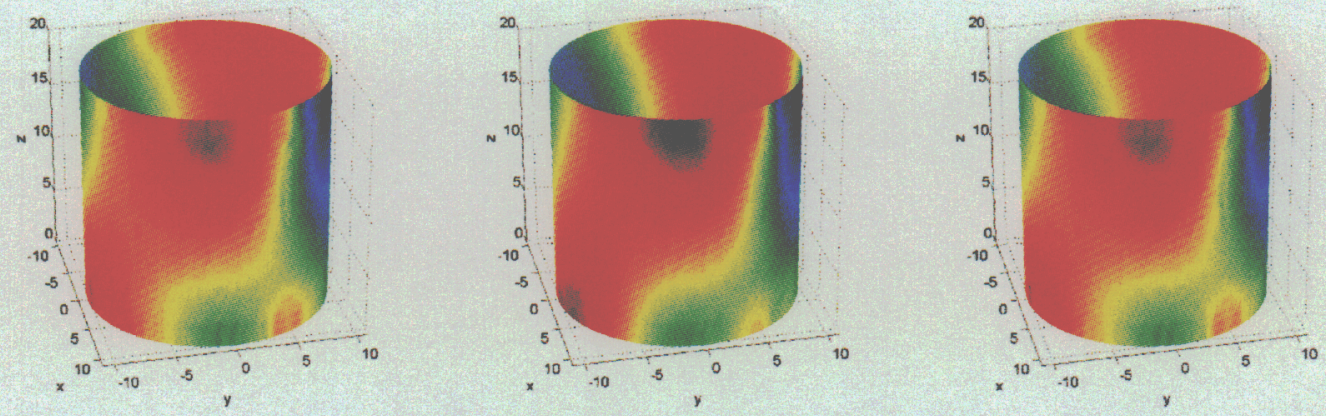

Figure 16: Contour Plots of the Radial Coordinates for the Experimental Data, Initial Finite Element Discretization and Final (Stress Free) Discretization

A three-dimensional contour map of the deformed radial coordinates for the experimental, initial (i.e. initial iteration) finite element analysis, and the final (i.e. converged) finite element analysis are shown in Figure 16.
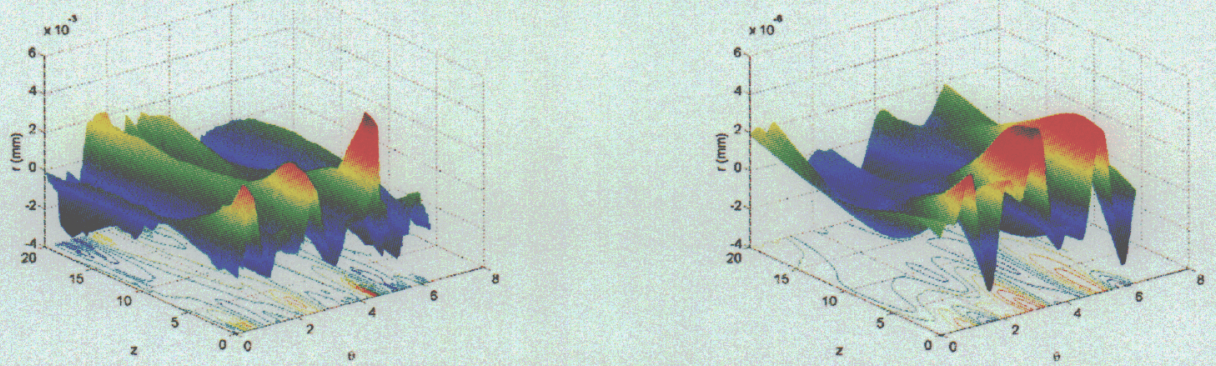

Figure 17: Contour Plots for the Error in Radial Coordinates of the initial and Final ( Stress Free) Discretization Referenced from the Experimental Data

In addition, Figure 17 shows the error in the deformed radial coordinate for the cases above. 
- Figure 18 depicts the initial configurations for the experimental data and the computed stress-free finite element data.
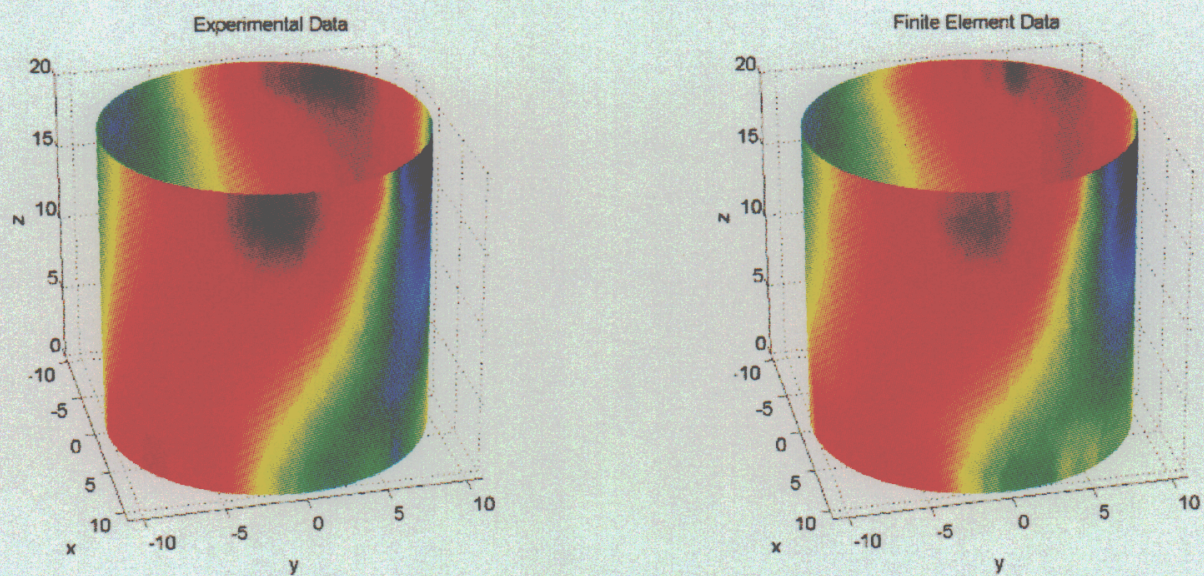

Figure 18: Contour Plots of the Radial Coordinates for the Initial Experimental and Stress Free Finite Element Data

To assess the uncertainty of the optically measured cylinder data to that of a "perfect" or "pure" cylinder and the stress-free configuration developed from the finite element analysis several scalar measures were utilized.

Table 2: Scalar Measures

\begin{tabular}{|l|c|c|c|}
\hline \multicolumn{1}{|c|}{ Description } & $\begin{array}{c}\mathrm{N}_{1} \\
(\mathrm{~mm})\end{array}$ & $\begin{array}{c}\mathrm{N}_{2} \\
(\mathrm{~mm})\end{array}$ & $\begin{array}{c}\varepsilon_{1} \\
(\mathrm{~N}-\mathrm{mm})\end{array}$ \\
\hline $\begin{array}{l}\text { Comparison of the experimental and finite } \\
\text { element data }\end{array}$ & $5.467 \times 10^{-6}$ & $1.158 \times 10^{-4}$ & -- \\
\hline $\begin{array}{l}\text { Comparison of the experimental and pure } \\
\text { cylinder data }\end{array}$ & $1.883 \times 10^{-2}$ & $5.156 \times 10^{-1}$ & -- \\
\hline $\begin{array}{l}\text { Stress-free finite element configuration to } \\
\text { initial experimental configuration }\end{array}$ & -- & -- & $7.632 \times 10^{0}$ \\
\hline $\begin{array}{l}\text { Stress-free finite element configuration to } \\
\text { initial pure cylinder configuration }\end{array}$ & -- & -- & $1.047 \times 10^{1}$ \\
\hline $\begin{array}{l}\text { Initial experimental configuration to pure } \\
\text { cylinder configuration }\end{array}$ & -- & -- & $2.217 \times 10^{0}$ \\
\hline
\end{tabular}

Table 2 lists the various scalars used, specifically an infinity norm defined as (3),

$$
\mathrm{N}_{1}=\max \left|R_{i j}^{\exp }-R_{i j}^{\text {fea }}\right| \text { or } \mathrm{N}_{1}=\max \left|R_{i j}^{\mathrm{exp}}-R_{i j}^{\text {pure }}\right|
$$

where $R_{i j}$ is the deformed radial coordinates associated with the discretization, the superscript $\exp$ denotes the experimental data, $f e a$ denotes the final finite element data, and pure denotes the data for a pure or perfect cylinder. The 2-norm defined as (4), 


$$
\mathrm{N}_{2}=\left[\sum_{i} \sum_{j}\left(R_{i j}^{\exp }-R_{i j}^{f e a}\right)^{2}\right]^{\frac{1}{2}} \text { or } \mathrm{N}_{2}=\left[\sum_{i} \sum_{j}\left(R_{i j}^{\exp }-R_{i j}^{\text {pure }}\right)^{p}\right]^{\frac{1}{2}}
$$

and the last scalar measure is an energy term, $\varepsilon_{1}$, which is defined as the work required (i.e., radial force ${ }^{*}$ radial distance) to deform a given initial configuration (e.g., the pure cylinder configuration) to another initial configuration (e.g., the stress-free finite element configuration).

\section{Conclusion}

The problem of metrology of non-rigid objects is complex. There is no pedigreed process as with rigid artifacts. The problem is encountered in normal industrial operations such as sheet metal processes but often addressed in non-generalizable methods. Further, it is encountered in precision engineering because, at some level of precision, everything is non-rigid.

This work provides new insight into the metrology of non-rigid objects. The research problem has been identified - a significant contribution in itself. A method to address the problem was formulated and demonstrated experimentally on a thin walled copper cylinder. The free shape was determined and several acceptance metrics computed.

Assessing the uncertainty in the non-rigid metrology process is a complicated issue. In addition to the typical uncertainty associated with the data acquisition there is uncertainty introduced with the computational analysis. Factors contributing to the overall uncertainty were identified and the relative contributions for many of them were quantified.

The results of this work have applications in several areas. At LLNL, the results can be used by EUVL for photomasks. NIF can also benefit by using the techniques to characterize KDP crystals. The original motivating problem, metrology of hemi-shells is possible, but not currently a priority with the customer. The clear posing of the research problem makes the issue more salient. This may lead to the desirable outcome of increased industrial research in the area.

This work was performed under the auspices of the U.S. Department of Energy by the University of California, Lawrence Livermore National Laboratory under Contract No. W-7405-Eng-48. 